\title{
Controle da Pressão Arterial e Fatores Associados em um Serviço Multidisciplinar de Tratamento da Hipertensão
}

\author{
Blood Pressure Control and Associated Factors in a Real-World Team-Based Care Center
}

\author{
Thiago Veiga Jardim, ${ }^{1,2}$ Ana Luiza Lima Souza, ${ }^{1,2}$ Weimar Kunz Sebba Barroso, ${ }^{1,2}$ Paulo Cesar B. Veiga Jardim ${ }^{1,2}$ \\ Universidade Federal de Goiás - Programa de Pós-Graduação em Ciências da Saúde, ${ }^{1}$ Goiânia, GO - Brasil \\ Universidade Federal de Goiás - Liga de Hipertensão Arterial, ${ }^{2}$ Goiânia, GO - Brasil
}

\section{Resumo}

Fundamento: Apesar de se recomendar a intervenção em equipe no tratamento da hipertensão, resultados dessa abordagem em ambientes do mundo real são escassos na literatura.

Objetivos: Apresentar os resultados de uma estratégia terapêutica baseada em equipe, de longo prazo, de pacientes hipertensos em um serviço de saúde.

Métodos: Dados de pacientes hipertensos acompanhados em um centro de tratamento multidisciplinar localizado na região centro-oeste do Brasil em junho de 2017 com pelo menos duas visitas de acompanhamento foram avaliados retrospectivamente. Dados antropométricos, pressão arterial (PA), tempo de acompanhamento, tratamento farmacológico, diabetes, estilo de vida foram coletados da última consulta. Valores de PA $<140$ x 90 mmHg em não diabéticos e $<130 \times 80 \mathrm{mmHg}$ em diabéticos foram considerados PA controlada. Um modelo de regressão logística foi construído para identificar variáveis independentemente associadas com o controle da PA. O nível de significância adotado foi de $p<0.05$.

Resultados: Foram incluídos 1548 pacientes, com média de acompanhamento de 7,6 \pm 7,1 anos. A maioria dos pacientes eram mulheres $(73,6 \% ; n=1139)$, com idade média de 61,8 anos. As taxas de controle da PA na amostra total, em não diabéticos e nos diabéticos foram $68 \%, 79 \%$, e 37,9\%, respectivamente. Diabetes associouse inversamente com controle da PA (OR 0,16; IC95\% 0,12-0,20; p<0,001), enquanto idade $\geq 60$ anos (OR 1,48; IC95\% 1,15-1,91; $p=0,003$ ) e sexo feminino (OR 1,38; IC95\% 1,05-1,82; $p=0,020$ ) apresentaram associação direta.

Conclusões: Uma taxa de controle de cerca de $70 \%$ foi encontrada em pacientes atendidos em um serviço multidisciplinar de tratamento da hipertensão. A fim de melhorar esses resultados, atenção deve ser dada a pacientes diabéticos, com idade menor que 60 anos e do sexo masculino. (Arq Bras Cardiol. 2020; 115(2):174181)

Palavras-chave: Hipertensão, Pressão Arterial/prevenção e controle, Exercício, Cooperação e Adesão ao Tratamento, Sedentarismo, Obesidade, Estilo de Vida.

\section{Abstract}

Background: Although team-based care is recommended for patients with hypertension, results of this intervention in a real-world setting are missing in the literature.

Objective: To report the results of a real-world long-term team-based care for hypertensive patients we conducted this study.

Methods: Data of hypertensive patients attending a multidisciplinary treatment center located in the Midwest region of Brazil in June 2017 with at least two follow-up visits were retrospectively assessed. Anthropometric, blood pressure (BP), follow-up time, pharmacological treatment, diabetes and lifestyle data were collected from the last visit to the service. BP values $<140 \times 90 \mathrm{mmHg}$ in non-diabetics and $<130 \times 80 \mathrm{mmHg}$ in diabetics were considered controlled. A logistic regression model was built to identify variables independently associated to BP control. Significance level adopted $p<0.05$

Correspondência: Thiago de Souza Veiga Jardim •

Universidade Federal de Goiás - Programa de Pós-Graduação em Ciências da Saúde - Primeira Avenida S/N Setor Leste Universitário, Goiânia, GO - Brasil E-mail: thiagoloirin@hotmail.com

Artigo recebido em 01/03/2019, revisado em 23/05/2019, aceito em 17/07/2019

DOI: https://doi.org/10.36660/abc.20180384 
Results: A total of 1,548 patients were included, with a mean follow-up time of $7.6 \pm 7.1$ years. Most patients were female $(73.6 \% ; n=1,139)$ with a mean age of $61.8 \pm 12.8$ years. BP control rates in all the sample, and in non-diabetics and diabetics were $68 \%$, $79 \%$, and $37.9 \%$, respectively. Diabetes was inversely associated with BP control (OR 0.16; 95\% Cl 0.12-0.20; $p<0.001)$ while age $\geq 60$ years $(\mathrm{OR} 1.48 ; 95 \% \mathrm{Cl}$ $1.15-1.91 ; p=0.003)$ and female sex $(O R 1.38 ; 95 \% \mathrm{Cl} 1.05-1.82 ; p=0.020)$ were directly associated.

Conclusions: a BP control rate of $70 \%$ was found in patients attending a multidisciplinary team care for hypertension. Further studies on male hypertensive, diabetic patients, younger than 60 years should be performed to confirm and improve these results. (Arq Bras Cardiol. 2020; 115(2):174-181)

Keywords: Hypertension; Blood Pressure/prevention and control; Exercise; Treatment Adherence and Compliance; Sedentarism; Obesity; Motor Activity; Life Style.

Full texts in English - http://www.arquivosonline.com.br

\section{Introdução}

A hipertensão (HTN) pode ser definida como elevação na pressão sanguínea detectada em duas ou mais leituras obtidas em duas ou mais ocasiões, ou uso de medicamentos anti-hipertensivos. ${ }^{1,2}$ Apesar do debate acerca dos limiares a serem adotados para definição da HTN, não há dúvida de que essa condição é um fator de risco cardiovascular e uma causa importante de incapacidade e morte..$^{3-5}$

Pressão arterial (PA) elevada é o fator de risco tratável mais importante de acidente vascular cerebral, fibrilação atrial e insuficiência cardíaca. Reduções na PA são efetivas para prevenir lesões nos órgãos alvo, eventos cardiovasculares e morte em condições clínicas variadas envolvendo diferentes níveis de PA, perfis de risco cardiovascular, e comorbidades. ${ }^{6,7}$ Apesar disso, a HTN não controlada continua uma situação muito prevalente em todo o mundo. ${ }^{8}$

Entre as estratégias que objetivam melhorar o controle da PA, intervenções em equipe têm se mostrado muito promissoras. ${ }^{9,10}$ Essas estratégias consistem em intervenções organizacionais centradas no paciente, multifacetadas, lideradas por equipes multidisciplinares, que objetivam melhorar a qualidade do cuidado à HTN. O tratamento da HTN em equipe inclui pacientes, profissionais da atenção primária, e outros profissionais, tais como cardiologistas, enfermeiros, farmacêuticos, médicos assistentes, nutricionistas, trabalhadores sociais, profissionais da saúde comunitária, entre outros. Esses profissionais se complementam, oferecendo apoio ao outro e dividindo responsabilidades. ${ }^{1}$

Apesar do cuidado em equipe ser recomendado para pacientes com HTN pela maioria das diretrizes, ${ }^{1,2,11,12}$ os resultados dessa intervenção em um ambiente real são escassos na literatura. Conduzimos o presente estudo com o objetivo de relatar os resultados de uma intervenção terapêutica multidisciplinar de longo prazo para pacientes com HTN, com foco na avaliação das taxas de controle da PA e fatores associados.

\section{Métodos}

Dados de todos os pacientes com HTN e idade de 18 anos, com pelo menos duas visitas de seguimento em um centro de tratamento multidisciplinar para HTN na região centro-oeste do Brasil em junho de 2017 foram avaliados retrospectivamente por conveniência.
A HTN foi definida de acordo com a $7 \underline{a}$ Diretriz Brasileira de Hipertensão Arterial: (1) PA no consultório $\geq 140 \times$ $90 \mathrm{mmHg}$; monitorização ambulatorial da PA $\geq 130 \times 80$ mmHg; (3) monitorização residencial da PA $\geq 135 \times 85$ mmHg. ${ }^{13}$ Pacientes em tratamento para HTN também foram considerados hipertensos.

O centro de tratamento em equipe multidisciplinar está em funcionamento por mais de 25 anos, e se dedica ao tratamento de HTN, à educação de profissionais da saúde e à pesquisa. Pacientes com diagnóstico recente de HTN e pacientes com dificuldade de controlar os níveis de PA foram encaminhados para o centro, e o número de pacientes incluídos no estudo foi 1701. A equipe multidisciplinar é composta por médicos (clínicos gerais, cardiologistas, endocrinologistas e nefrologistas), enfermeiros, nutricionistas, terapeutas ocupacionais, educadores físicos, psicólogos e musicoterapeutas. Com o objetivo de melhorar a adesão ao tratamento e reduzir perdas de acompanhamento, o intervalo máximo entre cada visita foi de três meses. O intervalo máximo entre duas consultas médicas foi de seis meses, e em relação a outros profissionais de saúde, não houve visitas de rotina, isto é, as consultas foram agendadas de acordo com as necessidades dos pacientes determinadas por avaliação clínica. Além disso, atividades educacionais e de promoção da saúde foram realizadas a cada duas semanas com os pacientes. ${ }^{14,15}$ Desde o início desse serviço multidisciplinar, as consultas foram registradas em um formulário padronizado. Todos os profissionais diretamente envolvidos no cuidado do paciente foram treinados rotineiramente para o preenchimento desse formulário, assegurando confiabilidade e reprodutibilidade dos dados ao longo dos anos. ${ }^{16,17}$

\section{Coleta de dados}

Foram coletados dados da última visita do paciente, independentemente da especialidade do profissional que o atendeu. Ainda, as datas da primeira consulta foram coletadas e usadas para calcular o período de acompanhamento (diferença entre a primeira e a última visita do serviço), em anos.

Os seguintes dados foram coletados dos prontuários médicos: sexo; idade: em anos, e avaliada pela diferença entre a data de nascimento e a data da última visita; dados antropométricos: peso, altura e Índice de Massa Corporal (IMC), calculado pela fórmula de Quetelet (IMC: peso em Kg/altura ${ }^{2}$ em metros). O estado nutricional foi classificado de acordo com o IMC, seguindo as definições 
da Organização Mundial de Saúde: sem sobrepeso (IMC < $25 \mathrm{~kg} / \mathrm{m}^{2}$ ); com sobrepeso (IMC $\geq 25 \mathrm{~kg} / \mathrm{m}^{2}$ e $<30 \mathrm{mg} / \mathrm{kg}^{2}$ ) e obeso (IMC $\geq 30 \mathrm{mg} / \mathrm{kg}^{2}$ ).

Pressão arterial: foram realizadas três ou mais medidas da PA, com intervalo mínimo de um minuto. Todas as medidas foram realizadas após cinco minutos de repouso, do membro superior, com o indivíduo sentado e braço apoiado. Foram usados manguitos de tamanho apropriado de acordo com o diâmetro do braço. Os valores médios das duas últimas medidas foram considerados para definição de controle da PA. As medidas foram realizadas com aparelhos oscilométricos (equipamentos semiautomáticos OMRON, modelo HEM-705 CP). Essa rotina foi adotada no serviço para evitar viés de observador.

Estilo de vida: tabagismo (fumante ou não fumante); consumo de bebidas alcóolicas (qualquer consumo relatado na última visita); atividade física de lazer (regular $\geq 3$ vezes por semana), irregular ( $<3$ vezes por semana) e sedentário (nenhuma atividade física).

Diabetes: definida seguindo-se as recomendações das diretrizes mais recentes da Sociedade Brasileira de Diabetes: ${ }^{18}$ (1) sintomas de poliúria, polidipsia, e perda de peso e glicemia casual (colhida em qualquer horário do dia, independente da última refeição realizada) $\geq 200 \mathrm{mg} / \mathrm{dL}$; (2) glicemia de jejum $\geq 126 \mathrm{mg} / \mathrm{dL}$; o diagnóstico deve ser confirmado repetindo-se a medida em outro dia em caso de pequenas elevações na glicemia; (3) glicemia de duas horas após o teste de tolerância à glicose oral $(75 \mathrm{~g}) \geq 200 \mathrm{mg} / \mathrm{dL}$. O tratamento ao diabetes registrado nos prontuários médicos também foi considerado como critério diagnóstico.

Tratamento medicamentoso: informação sobre tratamento farmacológico para HTN (se o paciente realizava bem como número de medicamentos).

\section{Definições de controle da PA}

Foram adotadas as recomendações da $7 \stackrel{\text { a }}{ }$ Diretriz Brasileira de Hipertensão Arterial $(2016)^{19}$ (valores de PA $<140 \times 90$ mmHg em não diabéticos e $<130$ x 80 mmHg em pacientes diabéticos) para análise do controle da PA.

\section{Serviço multidisciplinar}

Equipe médica: avaliou sintomas, estilo de vida, e medicamentos, realizou exame físico, analisou exames complementares e definiu o tratamento do paciente (prescrição de tratamentos farmacológico e não farmacológico, pedido de exames complementares, e agendamento de visitas de acompanhamento); encaminhou pacientes à emergência ou internação em caso de descompensação clínica aguda.

Enfermeiros: avaliaram sintomas, sinais vitais, estilo de vida e medicamentos; orientaram os pacientes quanto à adesão ao tratamento (farmacológico e não farmacológico); definiram intervalos de retornos com enfermeiro; e encaminharam pacientes para consulta médica se necessário em aspectos clínicos ou para manter um intervalo máximo de seis meses entre duas consultas médicas.

Nutricionistas: enfatizaram aspectos não medicamentosos do cuidado, especificamente a dieta; coletaram dados sobre dieta, avaliaram dados antropométricos e sinais vitais. A abordagem teve como objetivo orientações dietéticas, com ênfase em restrição de sal e prescrição dietética aos pacientes com diagnósticos específicos tais como diabetes e doença renal crônica.

Educadores físicos: promoveram atividade física em grupo para os pacientes (treinamento de resistência a exercício aeróbico) três vezes por semana, e enfatizaram a importância da prática regular de atividade física.

Os demais profissionais da saúde não realizaram visitas formais, e sim uma série de intervenções educacionais para promover a saúde dos pacientes. Os fisioterapeutas conduziram encontros periódicos agendados previamente ou se encontraram com os pacientes na sala de espera e discutiram medidas preventivas contra lesões e quedas. De maneira similar, os psicólogos e os terapeutas musicais atuaram principalmente na sala de espera, fornecendo orientações e intervenções com objetivo de reduzir estresse e melhorar o tempo de espera.

\section{Análise estatística}

A análise estatística foi realizada utilizando-se o programa STATA V14 (StataCorp., College Station, Texas, USA). O teste de Kolmogorov-Smirnov foi usado e confirmou que as variáveis contínuas apresentaram distribuição normal. As variáveis contínuas foram apresentadas em média e desvio padrão, e as variáveis categóricas em número e porcentagem. O teste $t$ não pareado foi usado para comparar as variáveis contínuas e o teste do qui-quadrado para comparar as variáveis categóricas. O modelo de regressão logística foi construído para identificar variáveis com associação independente com o controle da pressão sanguínea. Diabetes, idade $\geq 60$ anos, sexo feminino, consumo de bebida alcoólica, tabagismo, sedentarismo, tratamento medicamentoso, IMC, e tempo total de acompanhamento (anos) foram usados como preditores no modelo. O nível de significância adotado foi $\mathrm{p}<0.05$.

\section{Resultados}

Foram incluídos 1548 pacientes no estudo, correspondente a mais de $90 \%$ dos pacientes atendidos no serviço (153 não foram incluídos devido a dados faltantes na primeira ou na última consulta). O tempo médio de acompanhamento foi $7,6 \pm 7,1$ anos. A maioria dos pacientes eram mulheres (73,6\%; $n=1139)$, e a média de idade foi $61,8 \pm 12,8$ anos. As mulheres eram mais propensas a serem obesas e sedentárias, mas menos propensas a consumir bebida alcoólica e a fumar em comparação aos homens. Além disso, valores mais baixos de PA foram encontrados em mulheres em comparação a homens. Características da população do estudo, estratificadas por sex, são apresentadas na Tabela 1.

A taxa de controle da PA na população do estudo foi de $68 \%$, e esse valor foi mais alto quando somente pacientes não diabéticos foram considerados (79\%). Por outro lado, avaliando-se exclusivamente pacientes diabéticos, a taxa de controle da PA caiu para 37.9\%. A Figura 1 apresenta um resumo das taxas de controle da PA em nosso estudo.

Indivíduos com PA sob controle tendiam a ser do sexo feminino, mais velhas, com períodos mais longos de acompanhamento e IMC mais baixo em comparação 


\begin{tabular}{|c|c|c|c|c|}
\hline Fator & Total & Homens & Mulheres & Valor de $\mathrm{p}^{*}$ \\
\hline N & $1,548(100 \%)$ & $409(26.4 \%)$ & $1,139(73.6 \%)$ & \\
\hline Idade (anos) & $61,8( \pm 12,8)$ & $62,0( \pm 13,8)$ & $61,8( \pm 12,4)$ & 0,750 \\
\hline $\begin{array}{l}\text { Tempo total de acompanhamento } \\
\text { (anos) }\end{array}$ & $7,6( \pm 7,1)$ & $7,1( \pm 6,7)$ & $7,8( \pm 7,2)$ & 0,070 \\
\hline Altura $(\mathrm{m})$ & $1,58( \pm 0,09)$ & $1,67( \pm 0,08)$ & $1,55( \pm 0,07)$ & $<0,001$ \\
\hline Peso (kg) & $73,8( \pm 16,5)$ & $79,2( \pm 16,5)$ & $71,9( \pm 16,1)$ & $<0,001$ \\
\hline Índice de massa corporal $\left(\mathrm{kg} / \mathrm{m}^{2}\right)$ & $29,3( \pm 5,9)$ & $28,3( \pm 5,3)$ & $29,7( \pm 6,0)$ & $<0,001$ \\
\hline \multicolumn{5}{|l|}{ Estado nutricional } \\
\hline Sem sobrepeso & $350(22,6 \%)$ & $105(25,7 \%)$ & $245(21,5 \%)$ & 0,084 \\
\hline Com sobrepeso & $571(36,9 \%)$ & $174(42,5 \%)$ & $397(34,9 \%)$ & 0,006 \\
\hline Obeso & $627(40,5 \%)$ & $130(31,8 \%)$ & $497(43,6 \%)$ & $<0,001$ \\
\hline Primeira PA sistólica (mmHg) & $146,3( \pm 24,0)$ & $148,5( \pm 24,6)$ & $145,5( \pm 23,8)$ & 0,030 \\
\hline Primeira PA diastólica $(\mathrm{mmHg})$ & $85,5( \pm 16,0)$ & $87,2( \pm 15,6)$ & $84,9( \pm 16,1)$ & 0,014 \\
\hline Segunda PA sistólica (mmHg) & $144,5( \pm 23,1)$ & $146,8( \pm 23,1)$ & $143,7( \pm 23,0)$ & 0,018 \\
\hline Segunda PA diastólica $(\mathrm{mmHg})$ & $83,3( \pm 13,1)$ & $85,0( \pm 12,9)$ & $82,7( \pm 13,1)$ & 0,003 \\
\hline Terceira PA sistólica (mmHg) & $144,3( \pm 18,2)$ & $145,1( \pm 18,4)$ & $144,0( \pm 18,1)$ & 0,320 \\
\hline Terceira PA diastólica $(\mathrm{mmHg})$ & $83,2( \pm 10,2)$ & $84,4( \pm 10,4)$ & $82,8( \pm 10,1)$ & 0,009 \\
\hline PA sistólica média $(\mathrm{mmHg})^{\dagger}$ & $144,4( \pm 19,1)$ & $145,9( \pm 19,4)$ & $143,8( \pm 18,9)$ & 0,057 \\
\hline PA diastólica média $(\mathrm{mmHg})^{\dagger}$ & $83,3( \pm 10,6)$ & $84,7( \pm 10,7)$ & $82,8( \pm 10,6)$ & 0,002 \\
\hline Diabetes & $412(26,6 \%)$ & $113(27,6 \%)$ & $299(26,3 \%)$ & 0,590 \\
\hline Consumo de álcool & $206(13,3 \%)$ & $108(26,4 \%)$ & $98(8,6 \%)$ & $<0,001$ \\
\hline Tabagismo & $177(11,4 \%)$ & $73(17,8 \%)$ & $104(9,1 \%)$ & $<0,001$ \\
\hline \multicolumn{5}{|l|}{ Atividade física } \\
\hline Sedentarismo & $737(47,6 \%)$ & $172(42,1 \%)$ & $565(49,6 \%)$ & 0,009 \\
\hline Irregular & $231(14,9 \%)$ & $70(17,1 \%)$ & $161(14,1 \%)$ & 0,150 \\
\hline Regular & $580(37,5 \%)$ & $167(40,8 \%)$ & $413(36,3 \%)$ & 0,100 \\
\hline Tratamento farmacológico & $1,513(97,7 \%)$ & $399(97,6 \%)$ & $1,114(97,8 \%)$ & 0,770 \\
\hline Número de drogas anti-hipertensivas & $2,1( \pm 0,8)$ & $2,8( \pm 0,7)$ & $1,7( \pm 0,8)$ & 0,369 \\
\hline
\end{tabular}

Valores em média $( \pm D P)$ ou $n(\%)$; PA: pressão arterial; * teste $t$ não pareado para comparar variáveis contínuas e teste do qui-quadrado para comparar variáveis categóricas; significância estatística $a<0,05$. †média da segunda e da terceira leitura

àqueles com PA não controlada. Ainda, aqueles com PA controlada eram menos propensos a serem obesos, diabéticos e sedentários comparativamente àqueles com PA controlada. As características da população do estudo, estratificadas por controle de PA, são apresentadas na Tabela 2 .

O modelo de regressão logística multivariada usado para identificar variáveis independentemente associadas com o controle da PA nessa população mostrou que diabetes foi inversamente associado, enquanto idade $\geq 60$ anos e sexo feminino apresentaram associação direta com controle da PA (Tabela 3).

\section{Discussão}

Nós avaliamos os dados de mais de 1500 pacientes hipertensos acompanhamentos regularmente em um serviço de cuidado multidisciplinar para mostrar os resultados dessa abordagem em equipe em uma situação real. Todos os pacientes incluídos no estudo foram encaminhados para um centro especializado no tratamento da hipertensão e tiveram o tratamento coberto pelo Sistema Único de Saúde. Ainda, as características basais dos pacientes eram similares àquelas descritas no Registro Brasileiro de Hipertensão Arterial, ${ }^{20}$ mostrando a capacidade de se generalizar os resultados deste estudo. Quase 70\% dos pacientes apresentavam a PA controlada, e essa taxa subiu para $79 \%$ quando somente pacientes não diabéticos foram considerados. O controle da PA foi inversamente associado com diabetes e diretamente associado com idade $\geq 60$ anos e sexo feminino.

Estudos populacionais conduzidos no Brasil mostraram que as taxas de controle da PA variaram de 10,1\% a 57,6\%, dependendo da região do país e características dos pacientes. ${ }^{21}$ Nenhum desses estudos, no entanto, utilizou dados obtidos de centros de tratamento em equipe. Nossa taxa de controle da PA global (68\%) foi maior que as obtidas por tratamento convencionais. Em comparação às taxas de controle da PA relatadas em outros países de renda média como a África do Sul $(30 \text { e } 49 \%)^{22,23}$ e mesmo a um país de alta renda como os Estados Unidos (48\%), ${ }^{24}$ nós encontramos resultados melhores com intervenção baseada em equipe no estudo atual. 


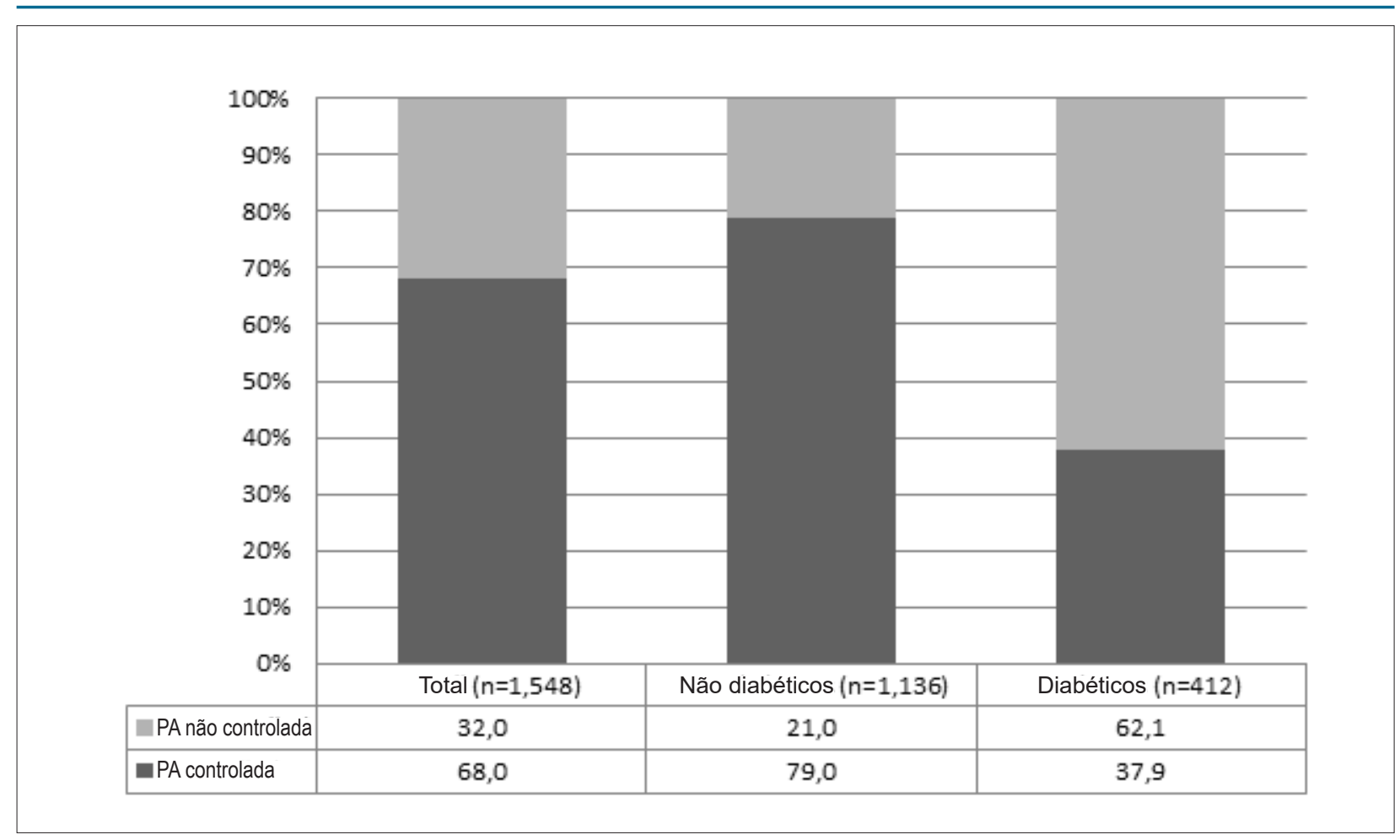

Figura 1 - Controle da pressão arterial na população total estudada, e em diabéticos e não diabéticos; Goiânia, Brasil. PA: pressão arterial; controle da pressão arterial: $P A<140 \times 90 \mathrm{mmHg}$ em não diabéticos e $<130 \times 80 \mathrm{mmHg}$ em diabéticos.

O controle da PA em pacientes com HTN e diabetes é um desafio; as taxas de controle são geralmente mais baixas em comparação a de pacientes hipertensos sem diabetes. ${ }^{25}$ Além disso, pacientes diabéticos hipertensos têm maior chance de desenvolverem HTN resistente verdadeira. ${ }^{26}$ Somente 37,9\% de nossos pacientes diabéticos hipertensos apresentavam PA controlada, em oposição a $79 \%$ entre pacientes não diabéticos. Ainda, o diabetes apresentou associação inversa e independente com o controle da PA nesta abordagem terapêutica multidisciplinar.

Idade avançada tem sido associada com controle da PA em diferentes populações. ${ }^{22,27}$ Nossos resultados reforçam esse dado, uma vez que idade $\geq 60$ anos apresentou associação direta com o controle da PA. Além disso, nosso estudo é original ao apresentar uma associação entre idades mais avançadas e controle da PA em um abordagem de tratamento baseado em equipe.

Diferença na taxa de controle da PA entre sexo é controversa. Enquanto alguns estudos apontam que mulheres têm maior probabilidade de apresentarem HTN não controlada em comparação a homens, ${ }^{28}$ outros indicaram uma associação entre sexo feminino e controle adequado da PA. ${ }^{22}$ Em nosso serviço de equipe multidisciplinar, esta é a primeira vez que os resultados apontam para uma associação direta entre sexo feminino e melhores taxas de controle da PA. ${ }^{16,17}$

Ensaios clínicos randomizados são geralmente considerados a melhor evidência cientifica para se confirmar ou não a eficácia e a segurança de um tratamento. ${ }^{29,30}$ Uma vez que há evidências disponíveis e diretrizes recomendam tratamentos, é importante avaliar o desempenho dessas intervenções na vida real. A realidade do cuidado ao paciente em um ensaio clínico randomizado é diferente da prática clínica diária em muitos aspectos ${ }^{31}$ Nesse sentido, os resultados positivos apresentados aqui, particularmente considerando que nosso estudo foi realizado em um serviço de saúde público de um país com recursos limitados, reforçam a importância de uma abordagem baseada em equipe no tratamento da hipertensão.

O delineamento do estudo pode ser considerado como uma limitação, uma vez que realizamos um estudo retrospectivo unicêntrico, sem grupo controle. Apesar disso, todos os prontuários médicos são objetivos e seu preenchimento exaustivamente treinado nesse centro, contribuindo para a confiabilidade dos dados. Ainda, apesar de sabermos que o uso de um grupo controle seria mais apropriado, os resultados positivos aqui encontrados podem ser usados como base para estudos futuros e ajudar como informação aos profissionais de saúde sobre uma estratégia bem sucedida no manejo de pacientes com HTN.

Outra limitação potencial refere-se à avaliação da atividade física. Somente atividade física formal ou planejada - caminhada, corrida, ciclismo, natação, treinamento de força, etc. - foi considerada na análise em nosso estudo. Assim, as atividades físicas diárias não foram consideradas e, portanto, os resultados de estilo de vida sedentário podem ter sido superestimados.

Custos de implementação e manutenção devem ser considerados no tratamento em equipe da hipertensão. Apesar 


\begin{tabular}{|c|c|c|c|}
\hline Fator & PA controlada & PA não controlada & Valor de $\mathrm{p} \dagger$ \\
\hline N & 1,053 & 495 & \\
\hline Sexo feminino & $793(75,3 \%)$ & $346(69,9 \%)$ & 0,024 \\
\hline Idade (anos) & $62,8( \pm 13,1)$ & $59,8( \pm 11,9)$ & $<0,001$ \\
\hline Tempo de acompanhamento (anos) & $8,1( \pm 7,4)$ & $6,6( \pm 6,5)$ & $<0,001$ \\
\hline Altura (m) & $1,58( \pm 0,09)$ & $1,59( \pm 0,09)$ & 0,059 \\
\hline Peso (kg) & $72,4( \pm 16,6)$ & $76,9( \pm 15,9)$ & $<0,001$ \\
\hline Índice de massa corporal $\left(\mathrm{kg} / \mathrm{m}^{2}\right)$ & $28,9( \pm 5,8)$ & $30,4( \pm 6,0)$ & $<0,001$ \\
\hline \multicolumn{4}{|l|}{ Estado nutricional } \\
\hline Sem sobrepeso & $262(24,9 \%)$ & $88(17,8 \%)$ & 0,002 \\
\hline Com sobrepeso & $399(37,9 \%)$ & $172(34,7 \%)$ & 0,230 \\
\hline Obeso & $392(37,2 \%)$ & $235(47,5 \%)$ & $<0,001$ \\
\hline Primeira PA sistólica (mmHg) & $138,8(19,8)$ & $162,2(24,4)$ & $<0,001$ \\
\hline Primeira PA diastólica $(\mathrm{mmHg})$ & $80,6(14,7)$ & $95,9(13,4)$ & $<0,001$ \\
\hline Segunda PA sistólica (mmHg) & $137,0(18,8)$ & $160,3(23,4)$ & $<0,001$ \\
\hline Segunda PA diastólica $(\mathrm{mmHg})$ & $77,7(9,9)$ & $95,2(11,0)$ & $<0,001$ \\
\hline Terceira PA sistólica (mmHg) & $139,4(15,8)$ & $154,7(18,5)$ & $<0,001$ \\
\hline Terceira PA diastólica $(\mathrm{mmHg})$ & $79,58(7,8)$ & $91,3(10,1)$ & $<0,001$ \\
\hline PA sistólica média $(\mathrm{mmHg})^{\dagger \dagger}$ & $138,2( \pm 15,8)$ & $157,5( \pm 18,9)$ & $<0,001$ \\
\hline PA diastólica média $(\mathrm{mmHg})^{\dagger \dagger}$ & $78,6( \pm 7,7)$ & $93,2( \pm 9,0)$ & $<0,001$ \\
\hline Diabetes & $156(14,8 \%)$ & $256(51,7 \%)$ & $<0,001$ \\
\hline Consumo de álcool & $130(12,3 \%)$ & $76(15,4 \%)$ & 0,100 \\
\hline Tabagismo & $119(11,3 \%)$ & $58(11,7 \%)$ & 0,810 \\
\hline \multicolumn{4}{|l|}{ Atividade física } \\
\hline Sedentarismo & $479(45,5 \%)$ & $258(52,1 \%)$ & 0,015 \\
\hline Irregular & $163(15,5 \%)$ & $68(13,7 \%)$ & 0,370 \\
\hline Regular & $411(39,0 \%)$ & $169(34,1 \%)$ & 0,064 \\
\hline Tratamento farmacológico & $1,028(97,6 \%)$ & $485(98,0 \%)$ & 0,660 \\
\hline Número de drogas anti-hipertensivas & $3,00( \pm 0,81)$ & $2,81( \pm 0,76)$ & 0,432 \\
\hline
\end{tabular}

Valores dados em médias ( $\pm D P$ ) ou n (\%). *Controle da pressão arterial (PA) - $P A<140 \times 90 \mathrm{mmHg}$ em não diabéticos e $<130 \times 80 \mathrm{mmHg}$ em diabéticos. † teste t não pareado para comparações das variáveis contínuas e teste do qui-quadrado para comparações das variáveis categóricas; significância estatística a<0,05. łmédia da segunda e da terceira leitura

disso, a avaliação econômica dessa intervenção em países de alta renda mostrou que a abordagem multidisciplinar para melhorar o controle da PA é custo efetiva. ${ }^{32}$ As mesmas avaliações devem ser realizadas em países de renda média-baixa.

Dados os resultados positivos do presente estudo e estudos prévios envolvendo pacientes do mesmo centro de tratamento da HTN, ${ }^{14,16,17,33,34}$ o formato adotado em nosso serviço pode ser um modelo para outros centros de tratamento de pacientes com HTN que almejam implementar uma estratégia de tratamento em equipe.

\section{Conclusão}

No presente estudo, conduzido em um ambiente real, a taxa de controle da PA após uma abordagem baseada em equipe a pacientes hipertensos foi $70 \%$. A fim de melhorar esses resultados, atenção deve ser dada a pacientes diabéticos, com idade menor que 60 anos e do sexo masculino.

\section{Contribuição dos autores}

Concepção e desenho da pesquisa, Obtenção de financiamento, Redação do manuscrito e Revisão crítica do manuscrito quanto ao conteúdo intelectual importante: Jardim TSV, Souza ALL, Barroso WKS, Jardim PCBV; Obtenção de dados, Análise e interpretação dos dados e Análise estatística: Jardim TSV, Jardim PCBV

\section{Potencial conflito de interesses}

Declaro não haver conflito de interesses pertinentes.

\section{Fontes de financiamento}

O presente estudo foi financiado pela Universidade Federal de Goiás, Hospital das Clínicas da Universidade Federal de Goiás e Fundação de Apoio ao Hospital das Clínicas da UFG. 


\section{Artigo Original}

\begin{tabular}{lccc}
\hline Tabela 3 - Variáveis independentemente associadas com controle da pressão arterial (n=1548); Goiânia - Brasil & \\
\hline Variáveis & Odds Ratio & [Intervalo de confiança 95\%] & Valor de $p$ \\
\hline Diabetes & 0,15 & {$[0,11-0,20]$} & $<0,001$ \\
Idade $\geq 60$ anos & 1,45 & {$[1,13-1,90]$} & 0,005 \\
Sexo feminino & 1,36 & {$[1,09-1,88]$} & 0,022 \\
Consumo de álcool & 0,80 & {$[0,56-1,15]$} & 0,183 \\
Tabagismo & 1,25 & {$[0,80-1,80]$} & 0,330 \\
Sedentarismo & 0,78 & {$[0,60-1,02]$} & 0,053 \\
Tratamento farmacológico & 1,12 & {$[0,50-2,47]$} & 0,741 \\
Índice de massa corporal $\left(\mathrm{Kg} / \mathbf{m}^{2}\right)$ & 0,97 & {$[0,95-1,01]$} & 0,088 \\
Tempo de acompanhamento (anos) & 1,01 & {$[1,00-1,03]$} & 0,098 \\
Número de drogas anti-hipertensivas & 0,85 & {$[0,68-1,01]$} & 0,320 \\
\hline
\end{tabular}

\section{Vinculação acadêmica}

Não há vinculação deste estudo a programas de pósgraduação.

\section{Referências}

1. Whelton PK, Carey RM, Aronow WS, Casey DE Jr, Collins KJ, Dennison Himmelfarb C, et al. 2017 ACC/AHA/AAPA/ABC/ACPM/AGS/APhA/ASH/ ASPC/NMA/PCNA Guideline for the Prevention, Detection, Evaluation, and Management of High Blood Pressure in Adults: Executive Summary: A Report of the American College of Cardiology/American Heart Association Task Force on Clinical Practice Guidelines. Hypertension. 2018;71(6):12691324.

2. Mancia G, Fagard R, Narkiewicz K, Rendon J, Zanchetti A, Böhm M, et al. 2013 ESH/ESC Guidelines for the management of arterial hypertension: The Task Force for the management of arterial hypertension of the European Society of Hypertension (ESH) and of the European Society of Cardiology (ESC). Eur Heart J. 2013;34(28):2159-219.

3. Forouzanfar MH, Liu P, Roth GA, NgM, Biryukov S, Marczak L, et al. Global burden of hypertension and systolic blood pressure of at least 110 to 115 mm hg, 1990-2015. JAMA. 2017;317(2):165-82.

4. Lim SS, Vos T, Flaxman AD, Danaei G, Shibuya K, Adair-Rohani H, et al. A comparative risk assessment of burden of disease and injury attributable to 67 risk factors and risk factor clusters in 21 regions, 1990-2010: a systematic analysis for the Global Burden of Disease Study 2010. Lancet. 2012;380(9859):2224-60.

5. Blacher J, Levy BI, Mourad JJ, Safar ME, Bakris G. From epidemiological transition to modern cardiovascular epidemiology: hypertension in the 21st century. Lancet. 2016;388(10043):530-2.

6. Blood Pressure Lowering Treatment Trialists' Collaboration. Blood pressurelowering treatment based on cardiovascular risk: a meta-analysis of individual patient data. Lancet. 2014;384(9943):591-8.

7. Ettehad D, Emdin CA, Kiran A, Anderson SG, Callender T, Emberson J, et al. Blood pressure lowering for prevention of cardiovascular disease and death: a systematic review and meta-analysis. Lancet. 2016;387(10022):957-67.

8. Mills KT, Bundy JD, Kelly TN, Reed JE, Kearney PM, Reynolds K, et al. Global disparities of hypertension prevalence and control: a systematic analysis of population-based studies from 90 countries. Circulation. 2016;134(6):441-50.

\section{Aprovação ética e consentimento informado}

Este estudo foi aprovado pelo Comitê de Ética do Hospital das Clínicas da Universidade Federal de Goiás sob o número de protocolo 1822-180. Todos os procedimentos envolvidos nesse estudo estão de acordo com a Declaração de Helsinki de 1975, atualizada em 2013.

9. Proia KK, Thota AB, Njie GJ, Finnie RK, Hopkins DP, Mukhtar Q, et al. Team-based care and improved blood pressure control: a community guide systematic review. Am J Prev Med. 2014;47(1):86-99.

10. Carter BL, Rogers M, Daly J, Zheng S, James PA. The potency of team-based care interventions for hypertension. Arch Intern Med. 2009;169(19):1748-55.

11. Malachias MVB, Franco RJ, Forjaz CLM, Pierin AMG, Gowdak MMG, Klein MRST, et al. 7th Brazilian Guideline of Arterial Hypertension: chapter 6 non-pharmacological treatment. Arq Bras Cardiol. 2016;107(3 supl 3):30-4.

12. Leung AA, Nerenberg K, Daskalopoulou SS, McBrien K, Zarnke KB, Dasgupta K, et al. Hypertension Canada's 2016 Canadian Hypertension Education Program Guidelines for Blood Pressure Measurement, Diagnosis, Assessment of Risk, Prevention, and Treatment of Hypertension. Can J Cardiol. 2016;32(5):569-88.

13. Malachias MVB, Gomes MAM, Nobre F, Alessi A, Feitosa AD, Coelho EB. 7th Brazilian Guideline of Arterial Hypertension: chapter 2 - diagnosis and classification. Arq Bras Cardiol. 2016;107(3 supl 3):7-13.

14. Pereira DA, Costa NMSC, Sousa ALL, Jardim PCBV, Zanini CRO. The effect of educational intervention on the disease knowledge of diabetes mellitus patients. Rev Latino-Am Enfermagem. 2012;20(3):478-85.

15. Sousa AL, Jardim PC, Monego ET, Raimundo MS, Lopes KE, Coelho J, et al. Multidisciplinary experience in the approach to the hypertensive patient. Arq Bras Cardiol. 1992;59(1):31-5.

16. Jardim LM, Jardim TV, Souza WK, Pimenta CD, Sousa AL, Jardim PC. Multiprofessional treatment of high blood pressure in very elderly patients. Arq Bras Cardiol. 2017;108(1):53-9.

17. Jardim TV, Inuzuka S, Galvão L, Negretto LAF, Oliveira RO, Sá WF, et al. Multidisciplinary treatment of patients with diabetes and hypertension: experience of a Brazilian center. Diabetes Metab Syndr. 2018;10(3):1-8.

18. Milech A, Angelucci AP, Golbert A, Matheus A, Carrilho AJF, Ramalho AC, et al. Diretrizes da Sociedade Brasileira de Diabetes 2015-2016. São Paulo: A.C. Farmacêutica; 2016 
19. Malachias MVB, Amodeo C, Paula RB, Cordeiro Jr AC, Magalhães LBNC, Bodanese LC. 7th Brazilian Guideline of Arterial Hypertension: Chapter 8 - hypertension and associated clinical conditions. Arq. Bras. Cardiol. 2016;107(3 supl 3):44-8

20. Lopes RD, Barroso WKS, Brandao AA, Barbosa ECD, Malachias MVB, Gomes MM, et al. The First Brazilian Registry of Hypertension. Am Heart J. 2018 Nov;205:154-7.

21. Pinho NA, Pierin AMG. Hypertension control in brazilian publications. Arq. Bras. Cardiol. 2013;101(3):e65-e73.

22. Jardim TV, Reiger S, Abrahams-Gessel S, Gomez-Olive FX, Wagner RG, Wade A, et al. Hypertension management in a population of older adults in rural South Africa. J Hypertens. 2017;35(6):1283-9.

23. Folb N, Timmerman V, Levitt NS, Steyn K, Bachmann MO, Lund C, et al. Multimorbidity, control and treatment of noncommunicable diseases among primary healthcare attenders in the Western Cape, South Africa. S Afr Med J. 2015;105(8):642-7.

24. Gillespie CD, Hurvitz KA, Centers for Disease Control and Prevention (CDC). Prevalence of hypertension and controlled hypertension - United States, 2007-2010. MMWR Suppl. 2013;62(3):144-8.

25. Grossman A, Grossman E. Blood pressure control in type 2 diabetic patients. Cardiovasc Diabetol. 2017;16(1):3

26. Krieger EM, Drager LF, Giorgi DMA, Pereira AC, Barreto-Filho JAS, Nogueira AR, et al. Spironolactone versus clonidine as a fourth-drug therapy for resistant hypertension: the ReHOT randomized study (Resistant Hypertension Optimal Treatment). Hypertension. 2018;71(4):681-90.
27. Wang H, Zhang X, Zhang J, He Q, Hu R, Wang L, et al. Factors associated with prevalence, awareness, treatment and control of hypertension among adults in southern China: a community-based, cross-sectional survey. PLoS One. 2013;8(5):e62469.

28. Tipton AJ, Sullivan JC. Sex differences in blood pressure control: are T lymphocytes the missing link? Hypertension. 2014;64(2):237-9.

29. Harbour R, Miller J. A new system for grading recommendations in evidence based guidelines. BMJ. 2001;323(7308):334-6.

30. Stanley K. Design of randomized controlled trials. Circulation 2007;115(9):1164-9.

31. Nallamothu BK, Hayward RA, Bates ER. Beyond the randomized clinical trial: the role of effectiveness studies in evaluating cardiovascular therapies. Circulation. 2008;118(12):1294-303.

32. Jacob V, Chattopadhyay SK, Thota AB, Proia KK, Njie G, Hopkins DP, et al Economics of team-based care in controlling blood pressure: a Community Guide Systematic Review. Am J Prev Med. 2015;49(5):772-83.

33. Sousa AC, Jardim TV, Costa TO, Magalhães FG, Montelo MPM, Souza WKB, et al. Hypertensive diabetic patients: incidence of cardiovascular and renal outcomes in a historical cohort over 11 years. Diabetol Metab Syndr. 2017;9:98.

34. Barroso WKS, Jardim PCB, Jardim TSV, Souza CTS, Magalhães ALA, Ibrahim FM, et al. Hypertensive diabetic patients: guidelines for conduct and their difficulties. Arq Bras Cardiol. 2003;81(2):143-7. 